\title{
Novel Method for Generating Long-Range Correlations
}

\author{
HERNÁN MAKSE, ${ }^{a}$ SHLOMO HAVLIN, ${ }^{a, b}$ H. EUGENE STANLEY ${ }^{a}$ \\ and MOSHE SCHWARTZ ${ }^{c}$
}

\author{
${ }^{a}$ Center for Polymer Studies and Department of Physics, Boston University, Boston, MA USA \\ ${ }^{\mathrm{b}}$ Department of Physics, Bar-Ilan University, Ramat-Gan, Israel \\ ${ }^{c}$ School of Physics and Astronomy, Tel-Aviv University, Ramat-Aviv, Israel
}

\begin{abstract}
We propose an algorithm to generate a sequence of numbers with long-range power-law correlations which is well-suited for large systems. Starting with a set of random uncorrelated variables, we modify its Fourier transform to get a new sequence with longrange correlations. By mapping the variables to a one dimensional random walk problem we find analytical and numerical evidence of the existence of correlations in the whole system. We exemplify the method by applying it to a generalized percolation problem where the occupancy variables are generated from a long-range correlated sequence.
\end{abstract}

\section{Introduction}

Disorder in various real systems in nature is found to be usually long-range correlated. Numerical studies of such systems rely on the numerical methods used to generate the correlated noise. Recently [1] an algorithm to generate correlated noise has been proposed. Due to the finiteness of the system, and the discreteness of the lattice, the correlations were found to be valid only up to a certain range (usually one percent of the system size). Here we present a new algorithm that better takes into account the finite size conditions and discreteness of the lattice.

\section{The Method}

Consider a sequence of $N$ uncorrelated random numbers $\left\{u_{j}\right\}_{j=1, . ., N}$ defined in a one-dimensional lattice. with the correlation function given by

$$
\left\langle u_{j} u_{j+n}\right\rangle=\delta_{n, 0}
$$

Our goal is to modify this sequence of numbers to generate a new one, $\left\{g_{j}\right\}_{j=1, . ., N}$, with long-range power-law correlations given by

$$
C_{n} \equiv\left\langle g_{j} g_{j+n}\right\rangle \sim n^{-\gamma}
$$




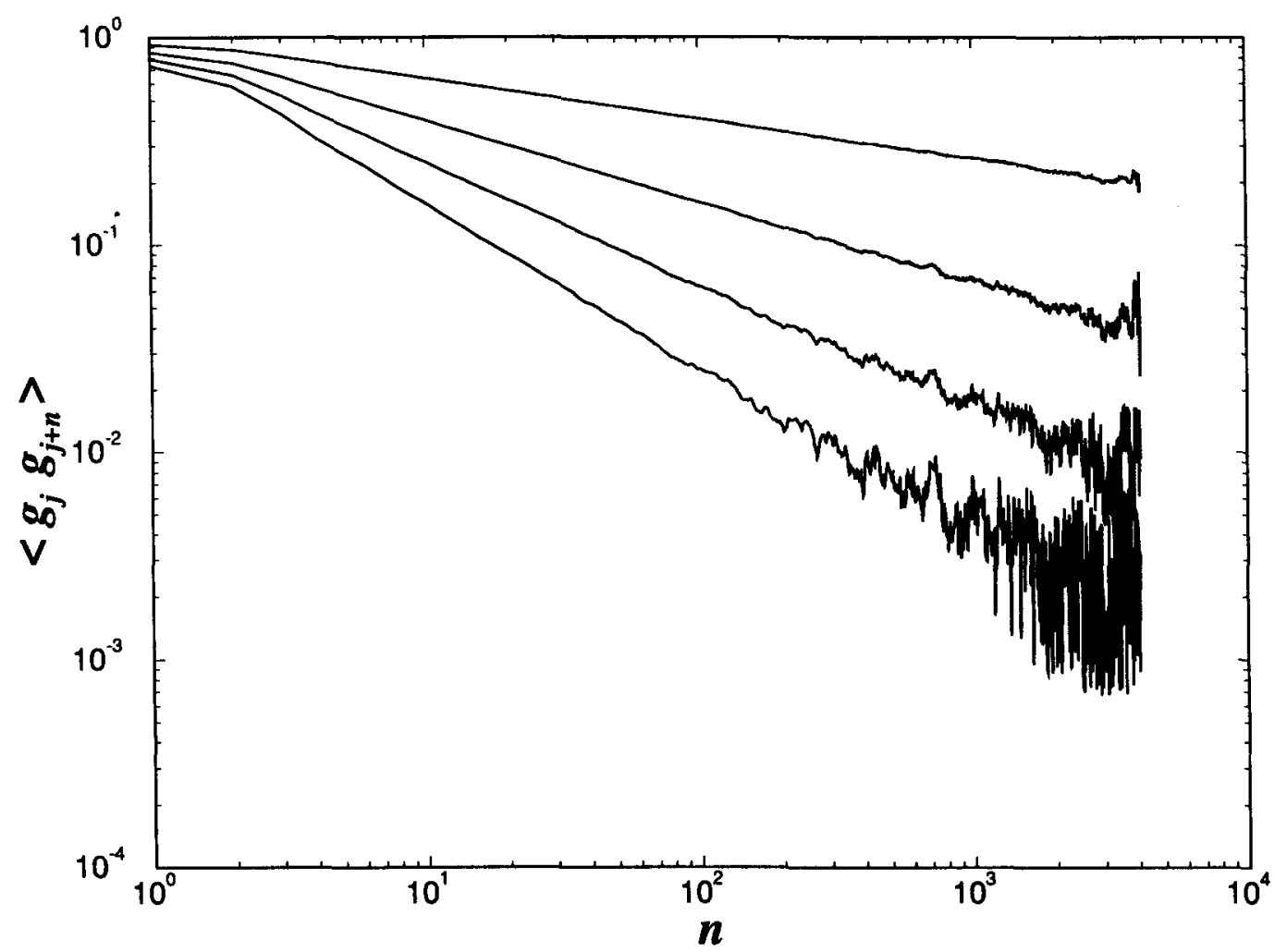

Fig. 1. A $\log -\log$ plot of the average correlation $\left\langle g_{j} g_{j+n}\right\rangle$ as a function of the distance $n$ between the sites for $N=4096$. Averages are taken over 500 realizations. Shown are results for different values of $\gamma=0.2,0.4,0.6$, and 0.8 (from top to bottom). The slopes of the linear fits are $0.19,0.37,0.57$ and 0.75 respectively.

We introduce a "response" function, $\phi_{j}$, that relates both sequences

$$
g_{j}=\sum_{k=1}^{N} \phi_{j-k} u_{k}
$$

By Fourier analyzing Eqs. (2) and (3) we find

$$
\hat{\Phi}_{q}=\sqrt{\hat{\mathcal{C}}_{q}} /\left|\hat{\mathcal{U}}_{q}\right|
$$

where $\hat{\Phi}_{q}, \hat{\mathcal{C}}_{q}$ and $\hat{\mathcal{U}}_{q}$ are the Fourier transforms of $\phi_{j}, C_{n}$ and $u_{j}$, respectively. 


\section{The algorithm}

The numerical algorithm we use to construct the correlated sequence, $\left\{g_{j}\right\}$, consists of the following steps:

- Generate the sequence, $\left\{u_{j}\right\}$, of uncorrelated random numbers and calculate its Fourier transform, $\hat{\mathcal{U}}_{q}$.

- Calculate the Fourier transform, $\hat{\mathcal{C}}_{q}$, of the desired correlation function $C_{n}$.

- Using Eq. (4) calculate the Fourier transform, $\hat{\Phi}_{q}$, of the "response" function and calculate the inverse Fourier transform to obtain $\phi_{j}$ in the real space.

- Substitute in Eq. (3) to get the correlated sequence, $\left\{g_{j}\right\}$.

In order to perform these steps numerically we take into consideration the corresponding periodic boundary conditions required by the discrete convolution theorem used to derive Eq. (4), and use the discrete Fourier transform:

$$
\hat{\mathcal{C}}_{q}=\frac{1}{N} \sum_{j=0}^{N-1} C_{j} e^{\frac{i 2 \pi q j}{N}}
$$

By calculating the correlation function $\left\langle g_{j} g_{j+n}\right\rangle$ averaging over the system, one can verify that long-range correlations have been constructed in the system (see Fig. 1).

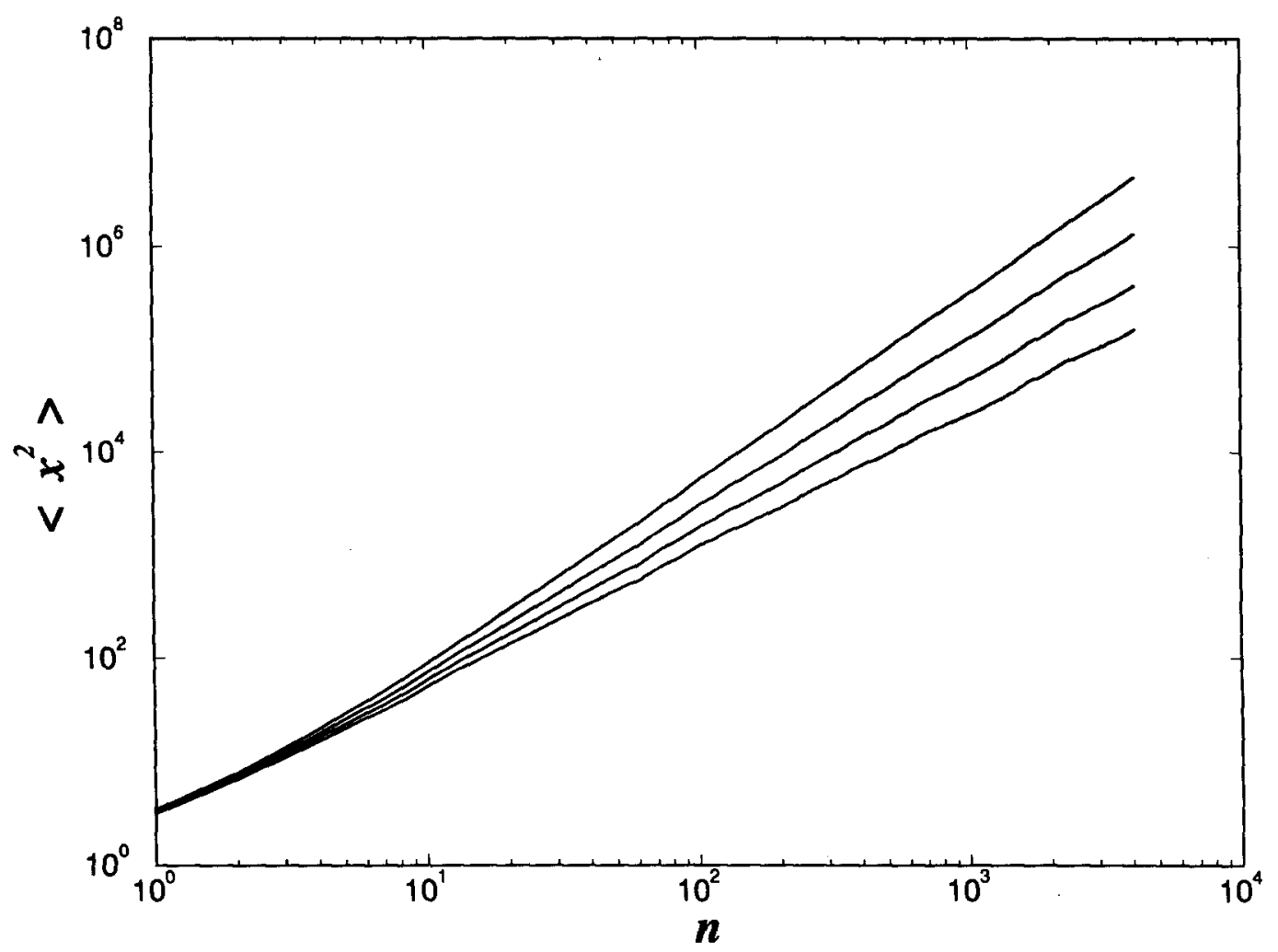

Fig. 2. Log-log plot of the mean square displacement for the same system of Figure 1, for $\gamma=0.2,0.4,0.6$ and 0.8 (from top to the bottom). The slopes for the linear fits are 1.81, 1.64, 1.46 and 1.31 respectively, in agreement with Eq. (7). 


\section{Generating Fractional Brownian Motion}

The sequence of correlated numbers, $\left\{g_{j}\right\}$, can be mapped to the sequence of steps of a random walk. We define the position of the walker at step $n$ by

$$
x_{n}=\sum_{j=1}^{n} g_{j}
$$

Each configuration $\left\{g_{j}\right\}_{j=1, . ., n} n \leq N / 2$, corresponds to an $n$-step random walk. When $\left\{g_{j}\right\}$ are correlated by a function given by Eq. (2), it can be shown that the dominant contribution to the mean square displacement, $\left.<x_{n}^{2}\right\rangle$ is

$$
\left\langle x_{n}^{2}\right\rangle \sim \begin{cases}n^{2-\gamma} & \text { if } \gamma<1 \\ n & \text { if } \gamma \geq 1\end{cases}
$$

Thus the long-range correlations lead to a power law behavior $\left\langle x_{n}^{2}\right\rangle \sim n^{2 H}$ for $\gamma<1$ with $H=1-\gamma / 2$ being the Hurst exponent. Therefore this procedure serves as a technique to generate fractional Brownian motion. On the other hand, the case $\gamma \geq 1$ corresponds to weak correlations and one recovers the uncorrelated random walk result, $\left\langle x_{n}^{2}>\sim n\right.$. Figure 2 shows the numerical results that confirm our calculations.

\section{The correlated percolation problem}

The properties of long-range correlated site percolation in the square lattice have been recently studied [2]. However, these studies were restricted to systems no larger than 104 x 104 sites. The method we present here allows us to study the correlated percolation problem for large systems.

The algorithm can be easily generalized to higher dimensions. In the two dimensional case the desired correlation function takes the form

$$
f(n, m) \sim\left(n^{2}+m^{2}\right)^{-\gamma / 2}
$$

and the correlated variables are defined in a square lattice $\left\{g_{k, j}\right\}$ with $k, j=1, \ldots N$.

In order to apply this procedure to the percolation problem we study the probability distribution $P(g)$ of the correlated sequence $\left\{g_{k, j}\right\}$. We find that when the uncorrelated variables $\left\{u_{k, j}\right\}$ are taken from a Gaussian distribution,

$\left\{g_{k, j}\right\}$ has also a Gaussian distribution. We next discretize the variables generating a sequence $\mu_{k, j}$, according to $\mu_{k, j}=\Theta\left(\theta-g_{k, j}\right)$ where $\theta$ is chosen to satisfy $p=\int_{-\infty}^{\theta} P(g) d g$, with $p$ the occupancy probability.

Figure 3 illustrates the results obtained for site percolation on a square lattice of $512 \times 512$ sites. Figures $3 \mathrm{a}$ and $3 \mathrm{~b}$ show the case of correlated occupancy variables (below and at the percolation threshold, respectively). Figures $3 \mathrm{c}$ and $3 \mathrm{~d}$ show the case of uncorrelated occupancy variables (below and at the percolation threshold, respectively). We see that the introduction of long-range correlations among the occupancy variables strongly affects the morphology of the system. In the correlated case the clusters look more compact than in the uncorrelated case. The lack of correlations in the uncorrelated case is seen from the presence of many small black holes inside the large clusters (Fig. 3d). Also, at small concentrations there are only small clusters (Fig. 3c) while for the correlated case, large clusters are present even at such low concentration (Fig. 3a).

This work is part of the Ph.D. Thesis of H. Makse. We wish to thank P. Jensen for help with the color graphics. 


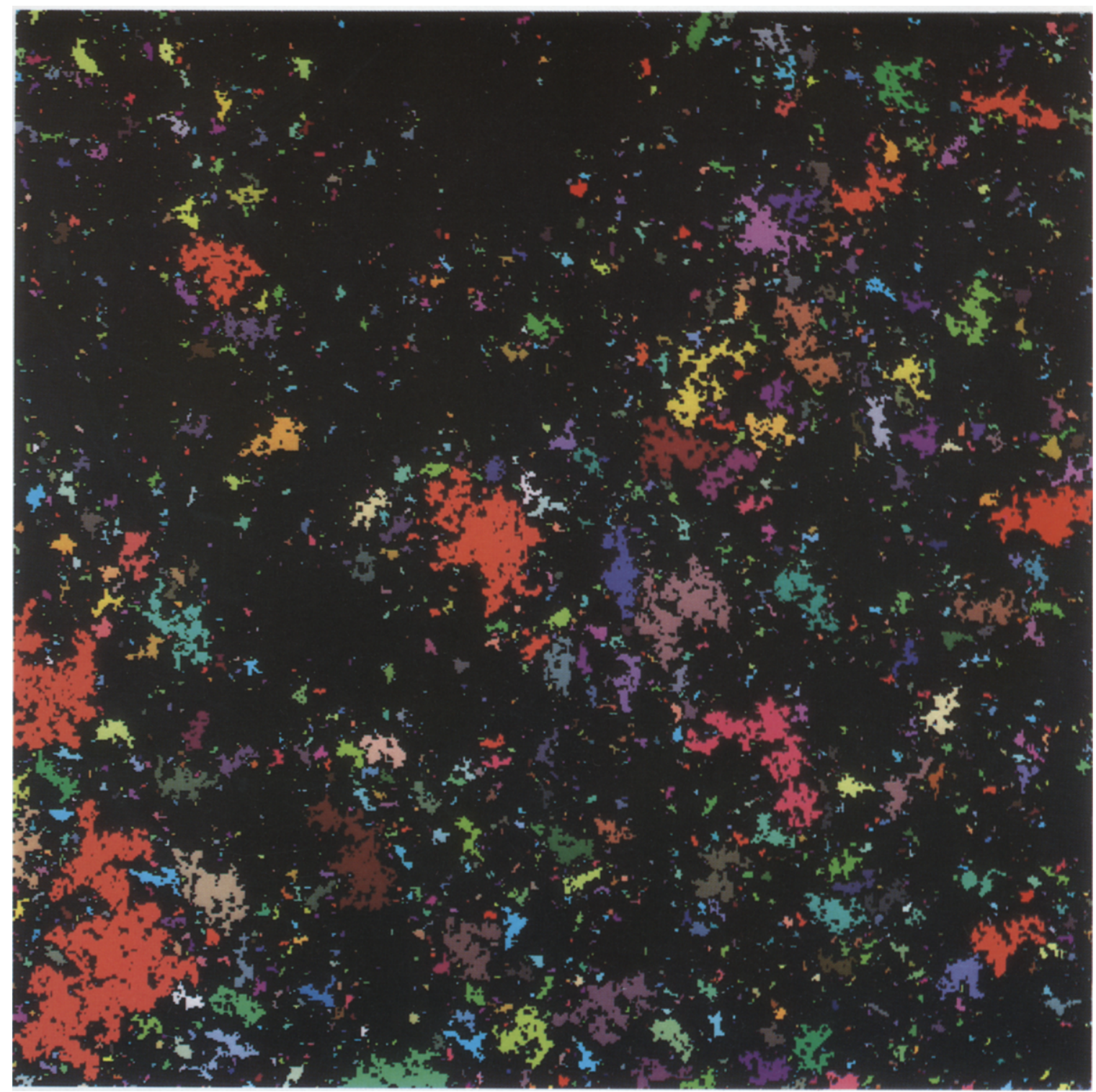

Fig. a

Fig. 3. Percolation in the square lattice of $512 \times 512$ sites for different degree of correlations and concentrations. Figures $\mathrm{a}$ and $\mathrm{b}$ correspond to the correlated case with $\gamma=0.4$ and concentration $p=0.2$ and $p_{c}$ respectively. Figures $\mathrm{c}$ and $\mathrm{d}$ correspond to the uncorrelated case with $p=0.2$ and $p_{c}$ respectively. Unoccupied sites are in black. Different colors represent different clusters. The spanning cluster at the critical concentration is in red. 


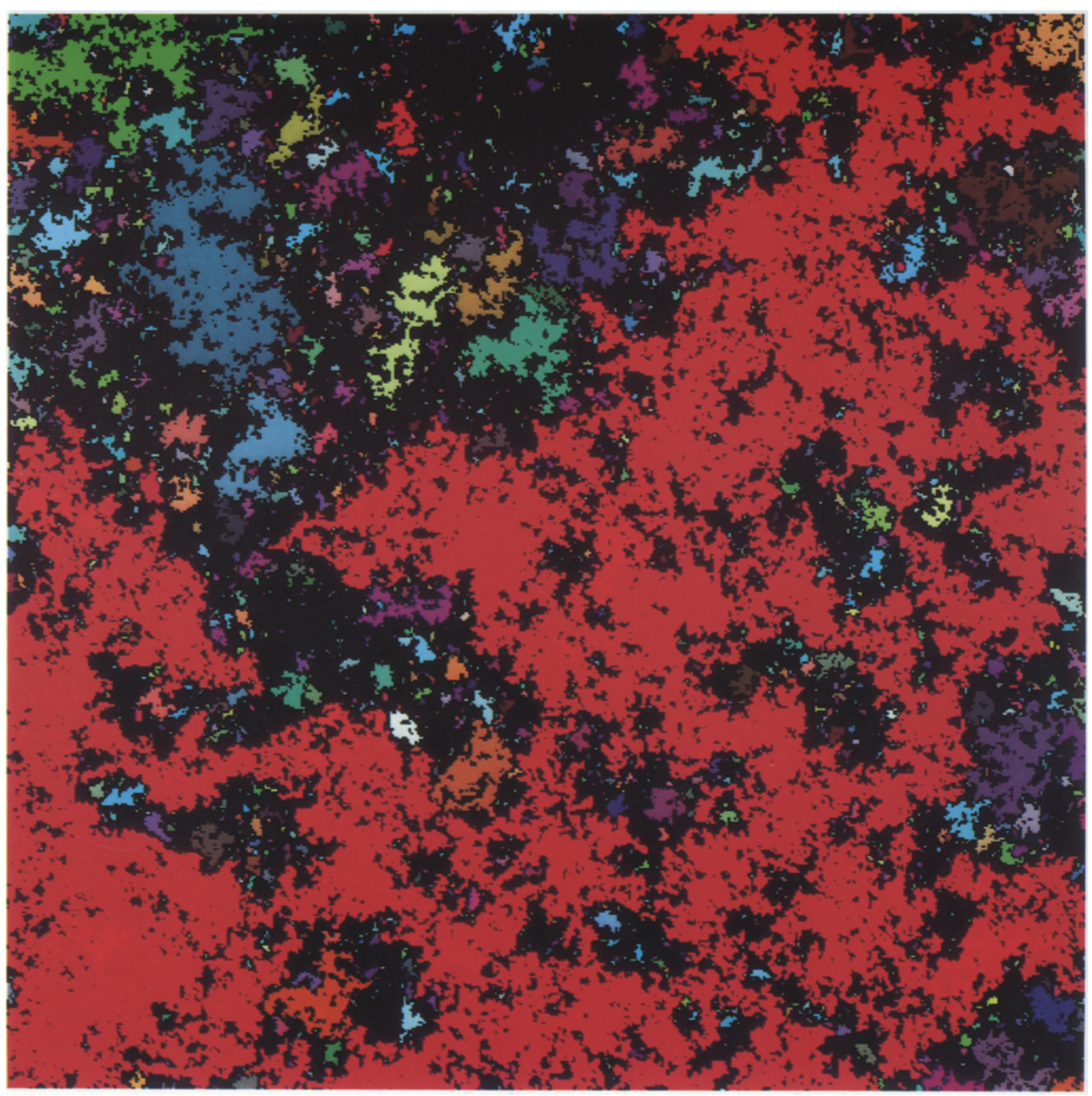

Fig. b 


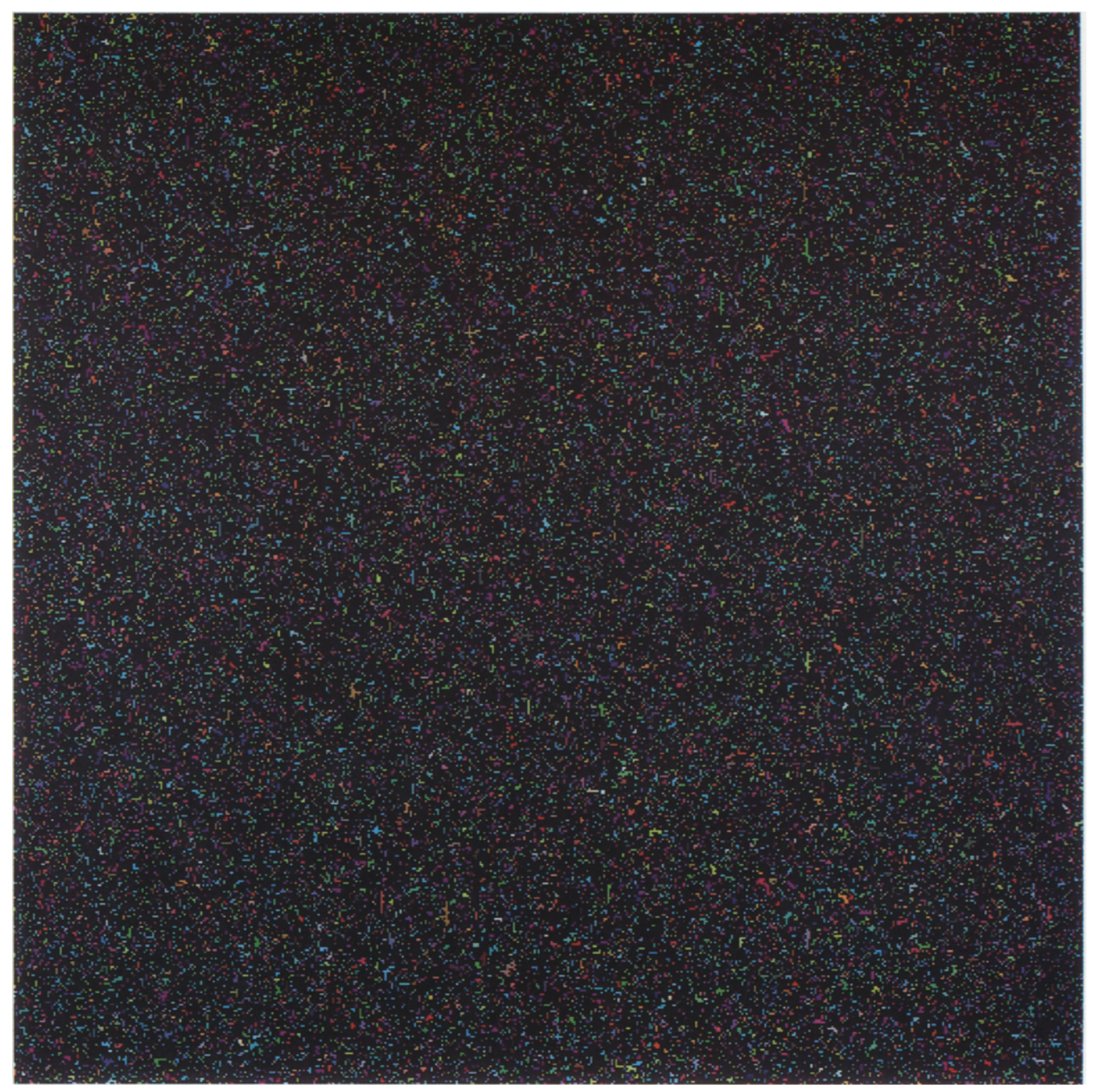

Fig. c 


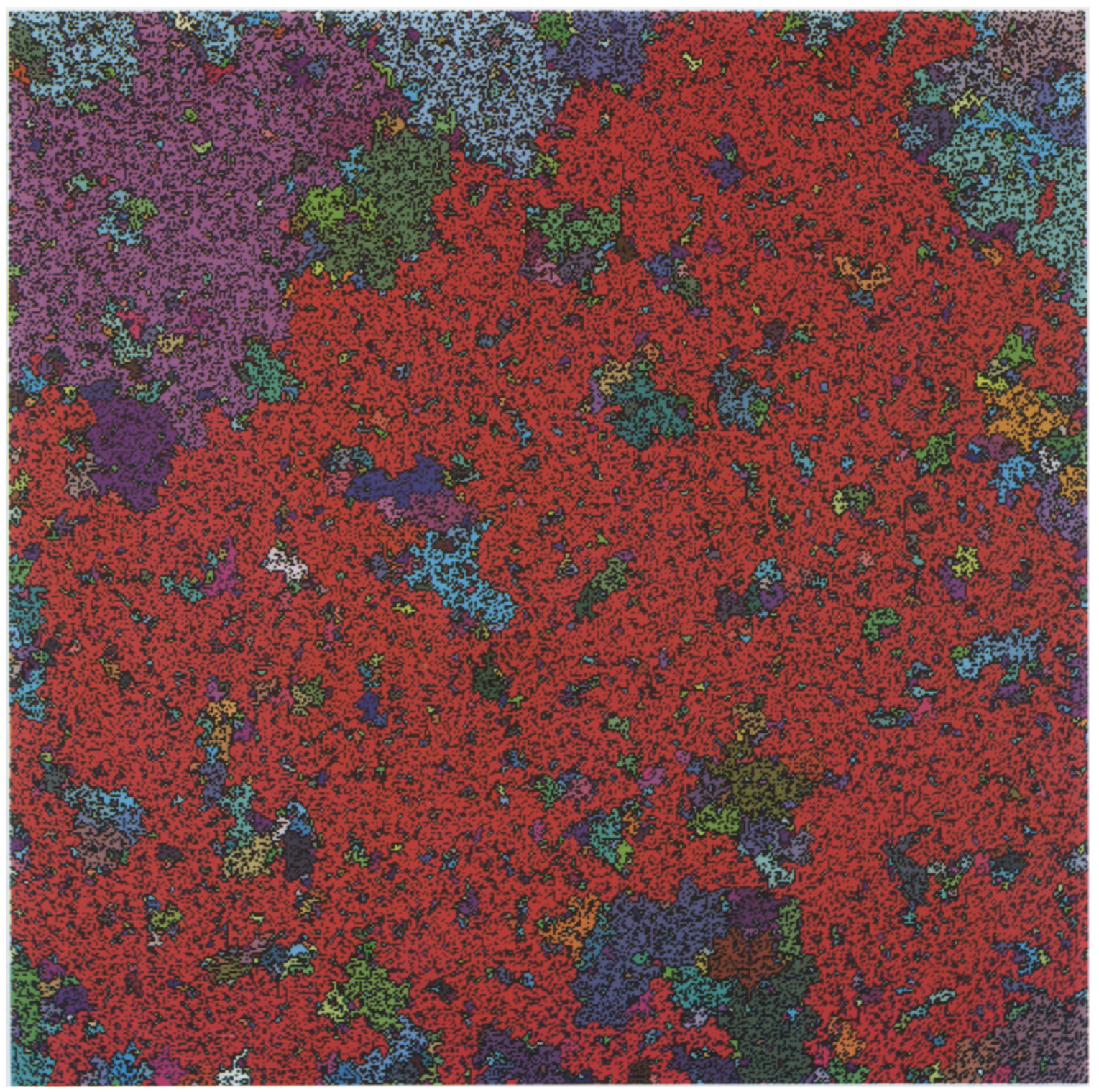

Fig. d 


\section{References}

[1] C. K. Peng, S. Havlin, M. Schwartz, and H. E. Stanley. Phys. Rev. A 44, 2239 (1991); S. Havlin, R. Selinger, M. Schwartz, H.E. Stanley and A. Bunde, Phys. Rev. Letters 61, 1438-1441 (1988). See also the explanation in Appendix A of A.-L. Barabasi and H. E. Stanley, Fractal Concepts in Surface Growth (Cambridge University Press, Cambridge, 1995). For a different method see also, R. F. Voss in Fundamental Algorithms in Computer Graphics, edited by R. Earnshaw (Springer-Verlag, Berlin, 1985), pp. 805-835.

[2] S. Prakash, S. Havlin, M. Schwartz, and H. E. Stanley. Phys. Rev. A 46, 1724 (1992). 